\title{
Molecular Approaches to Metastatic Colorectal Cancer: Better Diagnosis - Better Treatment?
}

\author{
Thomas Seufferlein $^{\mathrm{a}}$ Carolina Simões $^{\mathrm{b}}$ Fernando Kude $^{c}$ Thomas J. Ettrich ${ }^{\mathrm{a}}$ \\ ${ }^{a}$ Department of Internal Medicine I, Ulm University Hospital, Ulm, Germany; ${ }^{b}$ Hospital de Santa Maria, Centro

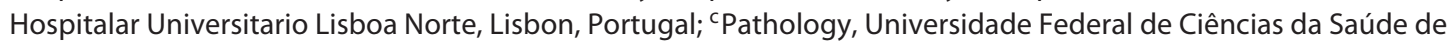 \\ Porto Alegre, Porto Alegre, Brazil
}

Keywords

Colorectal cancer · Molecular approach $\cdot$ Personalized medicine

\section{Abstract}

Molecular diagnostics of metastatic colorectal cancer (mCRC) are coming of age. Whereas for many years RAS mutations were the only relevant molecular alterations examined in mCRC, nowadays the BRAF, HER2, and microsatellite instability status of a given tumor is gaining importance, since it has not only prognostic relevance but opens new avenues for therapeutic approaches. This review summarizes the recent advances in molecular approaches to $\mathrm{MCRC}$ and their therapeutic consequences and discusses novel approaches for molecular diagnostics in $\mathrm{mCRC}$.

๑ 2019 S. Karger AG, Basel

\section{Introduction}

The treatment of metastatic colorectal cancer (mCRC) has improved over the recent years. This is mainly due to novel strategies, such as induction and maintenance treatments, the consequent screening for resectability in case of liver metastases and more efficacious treatment combinations, such as the FOLFOXIRI protocol either alone or in combination with the anti-VEGF antibody bevacizumab. Furthermore, there are novel chemotherapeutic agents for advanced lines of treatment, such as TAS102. Apart from surgery for liver metastases, there is an extensive "toolbox" that allows the treatment of localized disease, e.g., by microwave or radiofrequency ablation or stereotactic radiation. All these measures have made treatment of mCRC far more differentiated and have substantially improved outcomes. Nevertheless, recent advances in molecular classification and omics technologies open a new era of molecular based treatment of $\mathrm{mCRC}$.

\section{Left- and Right-Sided Primary CRCs and RAS Mutations}

Right-sided primary CRCs confer a worse prognosis in the metastatic setting as compared to left-sided tumors [1]. In one registry, the 5-year survival of mCRC rightversus left-sided primary was 1.4 versus $8.9 \%$, respectively. The "right colon" is hereby defined from the cecum to the splenic flexure. The precise reason for this finding is as yet unclear. However, there are marked differences between the right and the left colon in many aspects:

The frequency of CpG island methylator phenotype (CIMP)/microsatellite instability is markedly higher in the right colon. Wild-type KRAS and BRAF are increasingly found in the left colon, whereas the frequency of mutated KRAS (codon 12) and mutated BRAF (V600E) is declining towards the sigmoid colon [2].

There are also even differences in the microbiome between right-sided and left-sided CRCs. Whereas there is a higher abundance of Prevotella, Pyramidobacterium, Selemonas and Peptostreptococcus in the colon of right-

\section{KARGER}

(c) 2019 S. Karger AG, Basel
Prof. Dr. med. Thomas Seufferlein

Department of Internal Medicine I, Ulm University Hospital

Albert-Einstein-Allee 23

DE-89081 Ulm (Germany)

E-Mail thomas.seufferlein@uniklinik-ulm.de 
sided CRCs, the colon of left-sided CRCs exhibits a higher prevalence of Fusobacterium, Escherichia, Shigella and Leptotrichia [3]. These differences in the intestinal microbiota may also have relevance for the metastatic setting.

The right/left conundrum also extends to the therapeutic area. About $40 \%$ of all CRCs exhibit mutations in the RAS small G protein family. While we have known for quite some time that a RAS mutation in the primary tumor confers resistance to anti-EGFR antibodies, such as cetuximab and panitumumab, in the metastatic setting, a metaanalysis of several studies suggests that mCRCs with a right-sided primary tumor do also not respond to an antiEGFR treatment - at least as far as overall survival (OS) is concerned - even if the primary tumor has RAS wild-type status. In contrast, patients with $\mathrm{mCRC}$ and a left-sided primary tumor with a KRAS or NRAS and BRAF wild-type status do substantially benefit from the addition of antiEGFR antibodies to first-line chemotherapy [4].

\section{Is a RAS Wild-Type Tumor Always a RAS Wild-Type Tumor?}

Even if a (RAS wild-type) $\mathrm{mCRC}$ responds very well to anti-EGFR treatment in the first-line setting, we have learned that during anti-EGFR treatment, these patients acquire RAS and/or EGFR mutations. Interestingly, when anti-EGFR therapy is discontinued, RAS and EGFR relative mutant allele frequency decays exponentially with a half-life of 4.3 months. In these patients, antibodies such as cetuximab or panitumumab may be efficacious again after a longer anti-EGFR treatment-free interval [5]. However, not all of these patients respond again to an anti-EGFR treatment after a drug holiday: a small study investigated potential predictive biomarkers in blood [6]. The authors found that if there are still RAS mutations detectable in blood by analyzing circulating tumor DNA (ctDNA) after an anti-EGFR drug holiday of $\geq 4$ months, median progression-free survival (mPFS) and median OS (mOS) were significantly worse upon re-exposure to irinotecan plus cetuximab compared to patients without detectable RAS mutations in ctDNA (mPFS/mOS 1.9 and 5.2 months vs. 4.0 and 12.5 months, respectively). These data demonstrate that a re-challenge with cetuximab and irinotecan in patients with RAS/BRAF wild-type tumors and initial response to treatment only works when there is no RAS mutation detectable in the blood of patients.

\section{BRAF Mutations}

As stated above, the MAPK pathway is frequently mutated in CRC. Compared to RAS mutations, a smaller proportion of tumors, only $6-8 \%$ of mCRCs, exhibit muta- tions in the serine threonine kinase BRAF. Mutations in other RAF isoforms are even more rare. Amongst the mutations in BRAF, the V600E mutation belongs to the class I BRAF mutations that can signal as monomers independent of RAS activation and lead to high ERK activation [7]. This activation results in a particularly poor prognosis for patients with ${ }^{\mathrm{V} 600 \mathrm{E}} \mathrm{BRAF}-$ mutated mCRC. Interestingly, non- ${ }^{\mathrm{V} 600 \mathrm{E}} \mathrm{BRAF}$ mutations in $\mathrm{CRC}$ are not prognostic. ${ }^{\mathrm{V}} 600 \mathrm{E} \mathrm{BRAF}$ is also predictive: chemotherapy is not efficacious in these patients, and BRAF inhibitors - at least as single agents - also confer no benefit. There are few data mostly originating from subgroup analysis regarding treatment of patients with ${ }^{\mathrm{V} 600 \mathrm{E}} \mathrm{BRAF}$-mutated mCRC. A subgroup analysis of the TRIBE study demonstrated that patients with these tumors may benefit from a more intensive chemotherapy, in this case FOLFOXIRI plus bevacizumab compared to a chemotherapy doublet (FOLFIRI) plus bevacizumab [8]. A recent study examining the addition of panitumumab to the FOLFOXIRI protocol in patients with RAS wild-type mCRC showed very interesting response data in patients with ${ }^{\mathrm{V} 600 \mathrm{E}} \mathrm{BRAF}$-mutated tumors: here, the addition of panitumumab to a modified FOLFOXIRI protocol improved the overall tumor response rate from 22.2 to $85.7 \%$, a rate comparable to that observed in RAS/BRAF wild-type tumors. Alas, this marked improvement in tumor response did not translate into an improved PFS [9].

Based on promising preclinical data, the SWOG S1406 trial compared the combination of cetuximab, irinotecan, and the BRAF inhibitor vemurafenib with cetuximab plus irinotecan in patients with advanced mCRC. In total, 100 patients with BRAF-mutated tumors that had received at least 2 prior lines of treatment, but no anti-EGFR, antiBRAF, or anti-MEK therapy, were included. The trial met its primary endpoint with a median PFS of 4.3 months in the experimental arm and 2 months in the control arm (hazard ratio 0.48) [10]. Another interesting combination for patients with ${ }^{\mathrm{V} 600 \mathrm{E}} \mathrm{BRAF}$-mutated mCRC is based on the inhibition of several components of the EGFR-RASMEK-ERK pathway. The BEACON study examined the combination of binimetinib, a MEK inhibitor, encorafenib, a BRAF inhibitor, and cetuximab, an EGFRblocking antibody to avoid feedback activation of the EGFR. The trial has just reported the first safety and efficacy data from 29 patients demonstrating that the regimen is manageable with the most common grade $3 / 4$ adverse events being fatigue (13\%), anemia (10\%), increased creatine kinase (10\%), increased AST (10\%), and urinary tract infections (10\%). The overall response rate was $48 \%$. mPFS was 8 months and mOS was 15.3 months [11]. This is remarkable given the fact that all patients had experienced treatment failure with 1 or 2 regimens and the reported overall response rates in these patients are less than $10 \%$, the mPFS approximately 2 months, and the mOS between 4 and 6 months [10, 12-17]. 


\section{Novel Targets: HER2 and Gene Fusions}

Another growth factor receptor that is a potential target in CRC is HER2. The HERACLES trial examined a combination of trastuzumab plus lapatinib in patients with KRAS exon 2 wild-type mCRC that had received all approved treatments. The tumor had to have a 3+ HER2 score in more than $50 \%$ of cells by immunohistochemistry or 2+ HER2 score and a HER2:CEP17 ratio higher than 2 in more than $50 \%$ of cells by FISH [18]. $5 \%$ of the 914 patients screened fulfilled the criteria, and 27 patients were finally included into the trial. $30 \%$ of the treated patients achieved an objective response (ORR), 44\% of patients had stable disease. Treatment was well tolerated. In the MyPathway trial (phase II, multiple basket trial), 57 heavily pretreated patients ( $56 \%$ with $\geq 4$ previous therapeutic regimens) with a fresh HER2-positive tumor biopsy (immunohistochemistry $3+$ staining or $2+$ and HER2:CEP17 ratio higher than 2 by FISH or HER2 copy number $>6.0$ ) were included. The patients were treated with a combination of pertuzumab and trastuzumab [19]. $32 \%$ of patients archived an objective response. Disease control rate (DCR) was $44 \%$, PFS was 2.9 months, and OS was 11.5 months in the whole trial population $(n=57)$. In the subgroup of KRAS wild-type patients $(75.4 \%, n=43)$, ORR (40\%), DCR (56\%), PFS (5.3 months), and OS (14 months) were substantially improved compared to the subgroup with KRAS mutations $(22.8 \%, n=13$, ORR $8 \%$, DCR 8\%, PFS 1.4 months, OS 8.5 months). These results demonstrate that the concept of HER2 targeting in advanced $\mathrm{mCRC}$ is restricted to KRAS wild-type patients. Thus, analysis of the HER2 status is worthwhile in patients with advanced mCRC and KRAS wild type even if the cohort of patients eligible for anti-HER2 treatment is small.

A novel target not only in CRC are gene fusions. Fusions of the neurotrophic receptor tyrosine kinase genes 1,2 , and 3 result in activated tropomyosin receptor kinases $\mathrm{A}, \mathrm{B}$, and $\mathrm{C}$ (Trk $\mathrm{A}, \mathrm{B}$, and $\mathrm{C})$. The frequency of these fusions on $\mathrm{mCRC}$ is estimated to be $7 \%$. After initial screening by immunohistochemistry, RNA sequencing is performed to demonstrate the fusions of the respective genes. These fusions can be efficiently targeted with a small molecule inhibitor of Trk A, B, and C, larotrectinib. Patients with Trk fusions in their tumor do markedly benefit from treatment with larotrectinib with respect to tumor response and PFS [20].

\section{Immunooncology}

Treatment with immune checkpoint inhibitors has substantially improved the outcome of patients for a variety of tumors. In CRC, so far only the small subgroup of patients with mismatch repair deficient (dMMR)/highly microsatellite instable (MSI-H) tumors appears to benefit from a treatment with PD1 inhibitors, such as pembrolizumab or nivolumab, or a combination of nivolumab with the CTLA4 antibody ipilimumab. The group may comprise only $2-4 \%$ of patients with mCRC, but the markedly improved PFS and OS by checkpoint inhibitors in this group of patients [21] make it sensible to screen all patients with advanced mCRC for mismatch repair deficiency.

Treating patients with MSI-H/dMMR mCRCs may also be beneficial in earlier lines of treatment. A recent study presented data on the combination of nivolumab plus ipilimumab in the first-line treatment of patients with MSI-H/dMMR mCRCs [22]. 85\% of patients had a reduction in tumor burden from baseline, the 12 -month PFS rate was $77 \%$, and the 12 -month OS rate was $83 \%$. These data are very encouraging. There will be more information on this topic in due course since 2 other trials are close to reporting their results. One is comparing anti-PD1 treatment with conventional chemotherapy in the first-line treatment of MSI-H/ dMMR mCRCs (Keynote 177, ClinicalTrials.gov Identifier: NCT02563002), and the other is comparing mFOLFOX6 plus bevacizumab with atezolizumab or a combination of both in the same patient group (NRG-GI004/SWOG-1610, ClinicalTrials.gov Identifier: NCT02997228).

Approaches to make microsatellite stable/mismatch repair proficient tumors sensitive to immune checkpoint inhibition were so far less successful. The IMBlaze 370 study examined a combination of the antiPDL1 antibody atezolizumab with the MEK inhibitor cobimetinib and compared this combination with atezolizumab alone or regorafenib in patients with advanced CRC having received $\geq 2$ prior regimens of cytotoxic chemotherapy for metastatic disease. The primary endpoint was OS. The combination of atezolizumab plus cobimetinib did not improve OS or PFS compared to regorafenib or atezolizumab alone [23]. Another trial examined in a similar group of patients ( $n=180)$ with advanced CRC refractory to all available therapies the combination of durvalumab, an antiPD-L1 antibody, and the CTLA4 antibody tremelimumab compared to best supportive care [24]. There was no difference in PFS between the groups but a small difference in OS in favor of the treatment group (mOS durvalumab + tremelimumab 6.6 months vs. best supportive care 4.1 months; stratified hazard ratio 0.72 ; 90\% CI 0.54-0.97, $p=0.07$ ). In case of microsatellite stable mCRC, novel approaches, such as T-cell bispecific antibodies or CAR T cells, may be more promising therapeutic strategies, and trials investigating these approaches are under way. 
Table 1. CMS of colorectal cancer (adapted) $[25,31]$

\begin{tabular}{|c|c|c|c|c|}
\hline & CMS1, MSI immune & CMS2, canonical & CMS3, metabolic & CMS4, mesenchymal \\
\hline Prevalence & $14 \%$ & $37 \%$ & $13 \%$ & $23 \%$ \\
\hline $\begin{array}{l}\text { Genomic } \\
\text { aberrations }\end{array}$ & $\begin{array}{l}\text { Hypermethylation, } \\
\text { CIMP-high, MSI }\end{array}$ & $\begin{array}{l}\text { Copy number alterations, } \\
\text { chromosomal instability }\end{array}$ & $\begin{array}{l}\text { Mixed MSI status, } \\
\text { SCNA-low, CIMP-low }\end{array}$ & $\begin{array}{l}\text { Copy number alterations, } \\
\text { chromosomal instability }\end{array}$ \\
\hline KRAS mutation & Common & Common & Very common & Common \\
\hline BRAF mutation & Frequent & Very rare & Rare & Rare \\
\hline Gene expression & $\begin{array}{l}\text { Activation of immune } \\
\text { activation genes and } \\
\text { evasion pathways }\end{array}$ & $\begin{array}{l}\text { Epithelial differentiation; } \\
\text { WNT and MYC } \\
\text { activation }\end{array}$ & $\begin{array}{l}\text { Metabolic signatures, KRAS } \\
\text { activation }\end{array}$ & $\begin{array}{l}\text { Epithelial-to-mesenchymal } \\
\text { transition, TGF- } \beta \text { activation; } \\
\text { angiogenesis; matrix remodeling }\end{array}$ \\
\hline $\begin{array}{l}\text { Immune } \\
\text { signature }\end{array}$ & $\begin{array}{l}\text { Diffuse immune infiltra- } \\
\text { tion; high level of intratu- } \\
\text { moral cytotoxic T cells }\end{array}$ & Low immune activity & Low immune activity & $\begin{array}{l}\text { Complement-mediated } \\
\text { inflammation }\end{array}$ \\
\hline Clinical features & $\begin{array}{l}\text { Females; right-sided } \\
\text { lesions, high-grade }\end{array}$ & $\begin{array}{l}\text { Left-sided lesions, more } \\
\text { advanced stages }\end{array}$ & $\begin{array}{l}\text { Right-sided and early stages } \\
\text { frequent }\end{array}$ & $\begin{array}{l}\text { Diagnosed at more advanced stages } \\
\text { (III and IV) }\end{array}$ \\
\hline Prognosis & Poor prognosis after relapse & $\begin{array}{l}\text { Best overall survival after } \\
\text { relapse }\end{array}$ & $\begin{array}{l}\text { Relapse-free survival similar } \\
\text { to CMS2, but worse survival } \\
\text { after relapse }\end{array}$ & $\begin{array}{l}\text { Worse relapse-free and overall } \\
\text { survival }\end{array}$ \\
\hline
\end{tabular}

CMS, consensus molecular subtypes; CIMP, CpG island methylator phenotype; MSI, microsatellite instability; SCNA, somatic copy number alteration; TGF- $\beta$, transforming growth factor beta.

\section{Future Approaches}

The consensus molecular subtypes (CMS) of CRC (see Table 1) offer a molecular classification that divides CRC into 4 major subgroups [25]: CMS1, 14\% of all $\mathrm{CRC}$, has an immune signature and comprises MSI-H, CIMP-high, hypermutated tumors that are characterized by immune infiltration and activation as determined by a high level of intratumoral cytotoxic T cells. These tumors have a worse survival after relapse but may be particularly eligible for treatment with immune checkpoint inhibitors. CMS2, about 37\% of all CRCs, comprises the so-called canonical CRCs with a high prevalence of single copy number alterations (SCNA), WNT and MYC activation. CMS3, 13\% of all CRCs, has a metabolic signature that is characterized by a mixed MSI status, low SCNA prevalence, and low CIMP. Tumors are often KRAS mutated and exhibit metabolic deregulation as a potential therapeutic target. CMS2 and -3 tumors exhibit a low rate of intratumoral $\mathrm{T}$ cells and are unlikely to respond to PD1 inhibition, at least as a single agent. The last group, CMS4, comprises $23 \%$ of all CRCs, has a high prevalence of SCNA and is characterized by stroma infiltration, a high level of intratumoral TGF- $\beta$ activation and angiogenesis. The high stromal content classifies this CMA group as mesenchymal. Patients with CMS4 CRC have a worse relapse-free survival and OS [25-27]. Interestingly, the high intratumoral TGF- $\beta$ is likely to drive cytotoxic T-cell exclusion in these tumors and may make these tumors vulnerable to TGF- $\beta$ inhibition [28].

However, the CMS groups have been established in primary CRCs. Even though some features, such as MSI-H, are likely to be relevant for the metastatic situation, one must bear in mind that these signatures are not predictive of any kind of chemotherapy used for the treatment of mCRC.

However, molecular signatures may be able to guide treatment in the metastatic situation already now. In patients with mCRC and liver only metastases, we have currently mainly clinical parameters to determine resectability and/or recurrence of disease after resection, such as the FONG score [29]. A recent paper proposed a molecular subtyping system to predict recurrence and survival after resection of liver metastases. The authors differentiated 3 subtypes (canonical, immune, and stromal) and could attribute a risk classification to these subgroups [30]. The promising retrospective data in this article should be prospectively evaluated.

\section{Conclusion}

The treatment of mCRC has been substantially refined in the recent years. We can now differentiate several subgroups (left-/right-sided primary, RAS mutated, ${ }^{\mathrm{V}}{ }^{600} \mathrm{BR} A F$ mutated, MSI-H, HER2 overexpression, NTRK fusions), and more subgroups are likely to emerge in the near future. Identifying these subgroups is impor- 
tant since they require specific treatment strategies for optimal outcome. Thus, molecular diagnostics and molecular tumor boards are also getting more and more important in the area of $\mathrm{mCRC}$ to get a better diagnosis and a better treatment of this disease.

\section{Statement of Ethics}

The authors have no ethical conflicts to disclose.

\section{Disclosure Statement}

T.S. acted as consultant or received lecture fees from Bayer, BMS, Sanofi, Merck Serono, Roche, and Novartis outside the submitted work. One of his research projects is supported by Celgene.
T.J.E. received travel grants from IPSEN outside the submitted work. He acted as consultant for Bayer, BMS, Sanofi, Merck Serono, Roche, and Novartis outside the submitted work. He received lecture fees from Merck Serono, Sanofi, and Celgene. One of his research projects is supported by Servier. C.S. and F.K. have no possible conflicts of interest to declare.

\section{Funding Sources}

The authors have no funding sources to declare.

\section{Author Contributions}

T.S., T.J.E., C.S., and F.K. were responsible for conception, literature research, writing, and drafting the manuscript.

\section{References}

1 Price TJ, Beeke C, Ullah S, Padbury R, Maddern G, Roder D, et al. Does the primary site of colorectal cancer impact outcomes for patients with metastatic disease? Cancer. 2015 Mar;121(6):830-5.

2 Stintzing S, Tejpar S, Gibbs P, Thiebach L, Lenz HJ. Understanding the role of primary tumour localisation in colorectal cancer treatment and outcomes. Eur J Cancer. 2017 Oct; 84:69-80.

3 Gao Z, Guo B, Gao R, Zhu Q, Qin H. Microbiota disbiosis is associated with colorectal cancer. Front Microbiol. 2015 Feb;6:20.

4 Arnold D, Lueza B, Douillard JY, Peeters M, Lenz HJ, Venook A, et al. Prognostic and predictive value of primary tumour side in patients with RAS wild-type metastatic colorectal cancer treated with chemotherapy and EGFR directed antibodies in six randomized trials. Ann Oncol. 2017 Aug;28(8):1713-29.

5 Parseghian CM, Loree JM, Morris VK, Liu X, Clifton KK, Napolitano S, et al. Anti-EGFRresistant clones decay exponentially after progression: implications for anti-EGFR re-challenge. Ann Oncol. 2019 Feb;30(2):243-9.

6 Rossini D, Cremolini C, Conca E, Re MD, Busico A, Pietrantonio F, et al. Liquid biopsy to predict benefit from rechallenge with cetuximab (cet) + irinotecan (iri) in RAS/BRAF wild-type metastatic colorectal cancer patients (pts) with acquired resistance to firstline cet+iri: Final results and translational analyses of the CRICKET study by GONO. J Clin Oncol. 2018 May;36(suppl 15):1200712007.

7 Yaeger R, Corcoran RB. Targeting Alterations in the RAF-MEK Pathway. Cancer Discov. 2019 Mar;9(3):329-41.

8 Cremolini C, Loupakis F, Antoniotti C, Lupi C, Sensi E, Lonardi S, et al. FOLFOXIRI plus bevacizumab versus FOLFIRI plus bevacizumab as first-line treatment of patients with metastatic colorectal cancer: updated overall survival and molecular subgroup analyses of the open-label, phase 3 TRIBE study. Lancet Oncol. 2015 Oct;16(13):1306-15.
9 Geissler M, Klingler T, Riera-Knorrenschield J, Tannapfel A, Seufferlein T, Held S, et al. O024mFOLFOXIRI + Panitumumab versus FOLFOXIRI as first-line treatment in patients with RAS wild-type metastatic colorectal cancer $\mathrm{m}(\mathrm{CRC})$ : a randomized phase II VOLFI trial of the AIO (AIO-KRK0109). Ann Oncol. 2018 June;29(suppl 5).

10 Kopetz S, McDonough SL, Morris VK, Lenz H-J, Magliocco AM, Atreya CE, et al. Randomized trial of irinotecan and cetuximab with or without vemurafenib in BRAF-mutant metastatic colorectal cancer (SWOG 1406). J Clin Oncol. 2017 Feb;35(suppl 4): 520-520.

11 Kopetz S, Grothey A, Yaeger R, Cuyle P-JA, Huijberts S, Schellens JHM, et al. Updated results of the BEACON CRC safety lead-in: Encorafenib $(\mathrm{ENCO})+$ binimetinib (BINI) + cetuximab (CETUX) for BRAFV600E-mutant metastatic colorectal cancer (mCRC). J Clin Oncol. 2019 Feb;37(suppl 4):688-688.

12 Ulivi P, Capelli L, Valgiusti M, Zoli W, Scarpi E, Chiadini E, et al. Predictive role of multiple gene alterations in response to cetuximab in metastatic colorectal cancer: a single center study. J Transl Med. 2012 May;10(1):87.

13 Saridaki Z, Tzardi M, Sfakianaki M, Papadaki C, Voutsina A, Kalykaki A, et al. BRAFV600E mutation analysis in patients with metastatic colorectal cancer (mCRC) in daily clinical practice: correlations with clinical characteristics, and its impact on patients' outcome. PLoS One. 2013 Dec;8(12):e84604.

14 Seymour MT, Brown SR, Middleton G, Maughan T, Richman S, Gwyther S, et al. Panitumumab and irinotecan versus irinotecan alone for patients with KRAS wild-type, fluorouracil-resistant advanced colorectal cancer (PICCOLO): a prospectively stratified randomised trial. Lancet Oncol. 2013 Jul;14(8): 749-59.
15 Morris V, Overman MJ, Jiang ZQ, Garrett C, Agarwal S, Eng C, et al. Progression-free survival remains poor over sequential lines of systemic therapy in patients with BRAF-mutated colorectal cancer. Clin Colorectal Cancer. 2014 Sep;13(3):164-71.

16 Peeters M, Oliner KS, Price TJ, Cervantes A, Sobrero AF, Ducreux M, et al. Updated analysis of KRAS/NRAS and BRAF mutations in study 20050181 of panitumumab (pmab) plus FOLFIRI for second-line treatment (tx) of metastatic colorectal cancer (mCRC). J Clin Oncol. 2014 May;32(suppl 15):3568-3568.

17 Mitani S, Taniguchi H, Honda K, Masuishi T, Narita Y, Kadowaki S, et al. Analysis of efficacy and prognostic factors in second-line chemotherapy for BRAF V600E mutant metastatic colorectal cancer. Ann Oncol. 2017 Sept;28(suppl 5).

18 Sartore-Bianchi A, Trusolino L, Martino C, Bencardino K, Lonardi S, Bergamo F, et al. Dual-targeted therapy with trastuzumab and lapatinib in treatment-refractory, KRAS codon 12/13 wild-type, HER2-positive metastatic colorectal cancer (HERACLES): a proof-of-concept, multicentre, open-label, phase 2 trial. Lancet Oncol. 2016 Jun;17(6): $738-46$.

19 Meric-Bernstam F, Hurwitz H, Raghav KP, McWilliams RR, Fakih M, VanderWalde A, et al. Pertuzumab plus trastuzumab for HER2amplified metastatic colorectal cancer (MyPathway): an updated report from a multicentre, open-label, phase 2a, multiple basket study. Lancet Oncol. 2019 Apr;20(4):518-30.

20 Drilon A, Laetsch TW, Kummar S, DuBois SG, Lassen UN, Demetri GD, et al. Efficacy of Larotrectinib in TRK Fusion-Positive Cancers in Adults and Children. N Engl J Med. 2018 Feb;378(8):731-9.

21 Le DT, Uram JN, Wang H, Bartlett BR, Kemberling $\mathrm{H}$, Eyring AD, et al. PD-1 Blockade in Tumors with Mismatch-Repair Deficiency. N Engl J Med. 2015 Jun;372(26):2509-20. 
22 Lenz H-JJ, Van Cutsem E, Limon ML, Wong KY, Handlisz A, Aglietta M, et al. Durable clinical benefit with nivolumab (NIVO) plus low-dose ipilimumab (IPI) as first-line therapy in microsatellite instability-high/mismatch repair deficient (MSI-H/dMMR) metastatic colorectal cancer (mCRC). Ann Oncol. 2018 Oct;29(suppl 8).

23 Bendell J, Ciardiello F, Tabernero J, Tebbutt N, Eng C, Di Bartolomeo M, et al. Efficacy and safety results from IMblaze370, a randomised Phase III study comparing atezolizumab+cobimetinib and atezolizumab monotherapy vs regorafenib in chemotherapy-refractory metastatic colorectal cancer. Ann Oncol. 2018 June;29(suppl 5).
24 Chen EX, Jonker DJ, Kennecke HF, Berry SR, Couture F, Ahmad CE, et al. CCTG CO.26 trial: A phase II randomized study of durvalumab (D) plus tremelimumab (T) and best supportive care (BSC) versus BSC alone in patients (pts) with advanced refractory colorectal carcinoma (rCRC). J Clin Oncol. 2019 Feb; 37(suppl 4):481-481.

25 Guinney J, Dienstmann R, Wang X, de Reyniès A, Schlicker A, Soneson C, et al. The consensus molecular subtypes of colorectal cancer. Nat Med. 2015 Nov;21(11):13501356.

26 Kopetz S. New therapies and insights into the changing landscape of colorectal cancer. Nat Rev Gastroenterol Hepatol. 2019 Feb;16(2): 79-80.

27 Menter DG, Davis JS, Broom BM, Overman MJ, Morris J, Kopetz S. Back to the Colorectal Cancer Consensus Molecular Subtype Future. Curr Gastroenterol Rep. 2019 Jan;21(2):5.
28 Tauriello DV, Palomo-Ponce S, Stork D, Berenguer-Llergo A, Badia-Ramentol J, Iglesias $\mathrm{M}$, et al. TGF $\beta$ drives immune evasion in genetically reconstituted colon cancer metastasis. Nature. 2018 Feb;554(7693):538-43

29 Fong Y, Fortner J, Sun RL, Brennan MF, Blumgart LH. Clinical score for predicting recurrence after hepatic resection for metastatic colorectal cancer: analysis of 1001 consecutive cases. Ann Surg. 1999 Sep;230(3):309-18.

30 Pitroda SP, Khodarev NN, Huang L, Uppal A, Wightman SC, Ganai S, et al. Integrated molecular subtyping defines a curable oligometastatic state in colorectal liver metastasis. Nat Commun. 2018 May;9(1):1793.

31 Santos C, Sanz-Pamplona R, Nadal E, Grasselli J, Pernas S, Dienstmann R, et al. Intrinsic cancer subtypes-next steps into personalized medicine. Cell Oncol (Dordr). 2015 Feb; 38(1):3-16. 